\title{
Evaluation of a training package for the Malnutrition Universal Screening Tool (MUST)
}

\author{
S. H. Merriman, H. F. Scott, K. E. Paterson, R. Lee and E. A. Wainwright \\ Department of Nutrition and Dietetics, Norfolk and Norwich University Hospital, Colney Lane, Norwich NR4 7UY, UK
}

The National Institute of Health and Clinical Excellence (NICE) guidelines on nutrition support in adults ${ }^{(1)}$ recommend that all people in hospitals and Care Homes should be weighed and measured on admission. NICE advise screening should include BMI and unplanned weight loss. MUST is based on BMI and weight $\operatorname{loss}^{(2)}$. It is a validated, objective, screening tool that identifies adults who are underweight and at risk of malnutrition and provides guidelines for management.

Malnutrition is not always identified, therefore not treated ${ }^{(3)}$. The cost of malnutrition in the UK has been estimated to be $£ 7.3$ billion ${ }^{(4)}$ : more than twice the cost of obesity.

Our aim is that MUST is used in all settings in Norfolk. As a preliminary step we developed, delivered, and evaluated a pilot training package for six Social Services Care Homes. A 3-h training session that included formal teaching, practical work and group discussion was delivered twice to each Home. Post-training support was provided by email. Three months after completion of training Home Managers distributed questionnaires to all carers and caterers. Completed questionnaires and photocopies of all care plans and food charts were returned to the authors for audit and were cross-checked to ensure inter-rater reliability. All data was collated by an external auditor. The evaluation showed that staff considered they had received adequate training and that MUST was easy to use. Audit of care plans showed MUST was used accurately, and that appropriate actions were selected, implemented and monitored. The results of the care plan audit and comments made by staff have been used to develop a workbook for trainees.

Overall, $90 \%$ of carers were satisfied with their MUST training and found MUST easy to use. There was a high rate of accuracy (84\%) in calculation of MUST scores and appropriate care plans were developed from the guidelines (82\%). In general, providing snacks and fortified foods did not add to the workload but appropriate foods were reported to be available in only about half of the Homes.

Achieving our aim of MUST being used in all settings in Norfolk will involve training thousands of staff. This is not possible within our current workload. We are therefore developing a 'train the trainers' package. Social Services is generously funding a half-time post, for one year, to develop, deliver and evaluate this. They are also funding a researcher from the University of East Anglia to investigate anecdotal findings of a $30 \%$ reduction in the number of falls at Homes where MUST is used. The first tranche of 'train the trainer' has been completed and is being audited. Future developments will include developing e-learning and distance-learning packages.

1. National Institute of Health and Clinical Excellence (NICE) (2006) Nutrition support in adults - oral nutrition support, enteral tube feeding and parenteral nutrition (clinical guideline 32) London: National Institute for Health and Clinical Excellence (NICE).

2. British Association for Parenteral and Enteral Nutrition Malnutrition Advisory Group (2003) Nutritional screening of adults: a multidisciplinary responsibility. www.bapen.org

3. Abbasi AA \& Rudman D (1994) Nutrition Reviews 52 (4), 113-122.

4. Elia M, Stratton R, Russell C, Green C \& Pan F (2006) The cost of disease-related malnutrition in the UK and economic considerations for the use of oral nutritional supplements (ONS) in adults. www.bapen.org 